\title{
Prediction of admission to a low-resource sub-Saharan hospital by mental status, mobility and oxygen saturation recorded on arrival: a prospective observational study
}

\author{
Authors: Brian Kikomeko, ${ }^{A}$ George Mutiibwa, ${ }^{A}$ Pauline Nabatanzi, ${ }^{\mathrm{B}}$ Alfred Lumala ${ }^{\mathrm{C}}$ and John Kellett ${ }^{\mathrm{D}}$; on behalf of \\ the Kitovu Hospital Study Group
}

\section{Background}

The decision to admit patients to hospital in low-resource settings have been poorly investigated.

\section{Aim}

We aimed to determine the association of a disposition score determined on arrival with the decision subsequently made to admit or discharge the patient. The score awarded one point for altered mental status, one point for impaired mobility and one point for low oxygen saturation.

\section{Methods}

The mental status, mobility and oxygen saturation on arrival of 5,334 consecutive patients attending a combined emergency and outpatient department in a low-resource Ugandan hospital were recorded. Admission decisions were subsequently made independently by clinicians unaware to the score.

Results

Most patients ( $n=3,876 ; 73 \%$ ) had a disposition score of zero and only 25 of these patients $(0.6 \%)$ were subsequently admitted. A total of 646 (12.1\%) patients were admitted. Only 301 (5.6\%) patients had a score of 3 points and 263 (87.4\%) of these were admitted. The $\mathrm{C}$ statistic for the discrimination of the score for admission was 0.953 (95\% confidence interval 0.941-0.964).

Conclusion

In a low-resource setting, a simple score based on mental status, mobility and oxygen saturation identified outpatient and emergency department patients most and least likely to be subsequently admitted to hospital with a high degree of discrimination.

KEYWORDS: admission prediction, admission decisions, admission process, emergency admissions, triage scores

DOI: 10.7861/clinmed.2021-0325

Authors: ${ }^{\text {A }}$ research nurse, Kitovu Hospital, Masaka, Uganda; ${ }^{B}$ medical ward sister, Kitovu Hospital, Masaka, Uganda; ${ }^{C}$ medical

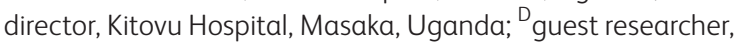
Hospital of South West Jutland, Esbjerg, Denmark

\section{Introduction}

Since hospitalisations comprise the largest component of healthcare expenditures, understanding the factors contributing to admission decisions is critical, especially in low-resource settings. ${ }^{1}$ Decisions to admit or discharge patients are usually made by whichever physician is encountered, and not by explicit evidence-based criteria. A National Institute for Health and Care Excellence (NICE) committee agreed that standardised criteria for admission are likely to be beneficial, but there is only evidence that they would benefit a limited number of conditions, such as upper gastrointestinal bleeding, community-acquired pneumonia and acute coronary syndromes. ${ }^{2}$

In the developed world, there is considerable variability in the number of emergency department patients who are admitted to hospital, ranging $12 \%$ to $48 \%{ }^{3}$. While the patients' clinical condition is the major influence on the likelihood of admission, non-medical factors may also contribute. ${ }^{1,4,5}$ These include patient income, insurance status, homelessness, spouse's health status, health literacy as well as access to care and other factors specific to individual healthcare systems. ${ }^{6}$ Most hospitalisations may be strongly or moderately influenced by one or more nonmedical factors. ' Little research has been carried out on what may influence or predict the decision to admit patients to hospital in low-resource settings.

Most validation studies of triage systems have used either the utilisation of resources or a patient outcome (such as mortality) as a proxy metric for the need for urgent treatment. ${ }^{7}$ However, the probability of admission should also largely capture the need for urgent care. Long waits before admission expose patients to worse outcomes, which might be reduced by an accurate early prediction of whether admission is likely. ${ }^{8,9}$ However, several studies conclude that clinical personnel cannot accurately predict admission using their judgement alone, and objective variables (such as age, triage category and physiological early warning scores) have been used to estimate admission probability. ${ }^{10-14}$ Some of these admission prediction tools require bespoke computer programmes or information that is be immediately available when the patient presents. $^{15-17}$

As part of an ongoing quality improvement project, the Kitovu Hospital Study Group used inpatient data to derive and validate a simple disposition score that potentially could supplement triage processes (such as the South African Triage System). The score 
awards one point for each of altered mental status, impaired mobility and low oxygen saturation; a patient with a score of zero points has an in-hospital mortality of $\leq 1 \%{ }^{18}$ Currently, Kitovu Hospital has not adopted a formal triage process. To determine if the disposition score would be helpful, practical, affordable and sustainable before adopting it as part of the hospital's care processes, researchers recorded the score on every patient when they first arrived at the hospital's outpatient and emergency departments. Here, we report the association of the score recorded when the patient was first encountered with the admission decisions made independently by clinicians who had no sight or knowledge of the score.

\section{Methods}

Aim

We aimed to determine the association of a disposition score recorded at presentation with the independent decision made to admit the patient to hospital.

\section{Setting}

This prospective observational non-interventional study was performed in the emergency and outpatient departments of Kitovu Hospital, which has 248 beds ( 50 medical and 35 surgical) and is located near Masaka, Uganda. It is a private not-for-profit (PNFP) hospital, accredited by the Uganda Catholic Medical Bureau. The average charge for a medical admission is US\$30, and the income of most patients attending the hospital is below the average Ugandan annual income of US $\$ 1,035 .{ }^{19}$

Currently, the hospital has no full-time specialist physicians and most emergency medical care is provided by recently qualified doctors (within 3 years of graduation) assisted by clinical officers (non-physician clinicians). ${ }^{20}$ Like many hospitals in low-resource settings, the distinction between outpatient and emergency patients is poorly defined. Many patients attend because of an acute or urgent problem, which makes maintaining precise appointment times for patients who are regular attenders with chronic problems impractical. Therefore, patients are seen on an informal 'first come, first served' basis. Although there is currently no formal triage process, patients who are in pain or severely injured are seen as quickly as possible. The emergency and outpatient departments are located beside each other and share clinical staff who move between them as needed. Patients enter both departments through a common lobby. The emergency department is open 24 hours a day and the outpatient department from $9 \mathrm{am}$ to $5 \mathrm{pm}$. After arrival, patients are directed to either the emergency department or the outpatient clinic by an informal process, depending on patient wishes, staff availability and their judgement, crowding, time of day etc. During the day, the combined departments are staffed by at least two clinical officers and a doctor and, at night, one doctor is first on-call and supported by two others who are second and third on-call. Twice a week, there are outpatient clinics attended by visiting consultant specialists.

\section{Participants and study process}

Participants were all patients aged 14 years or older who consecutively attended either the outpatient or emergency department from 23 November 2020 to 30 March 2021. During the day, a dedicated researcher entered patients' age, gender, presenting mental status, mobility, heart rate and oxygen saturation into an MS Excel database (Version 2102; Microsoft, Redmond, USA). At night, this information was recorded by the nurse on duty and entered in the database by the researcher the following morning. Data were collected on all patients on arrival. Therefore, frequent attenders may have been entered multiple times. The subsequent immediate disposition of each patient was also recorded (admitted, discharged or died while in the emergency department). The clinical staff caring for the patient had no access or knowledge of the data collected or the study purpose and all their management decisions were made independently of it.

There was no formal training of the study's researchers (or of the nurses who assisted them when they were not available) on how to recognise altered mental status or impaired mobility. Patients were considered to have altered mental status if they could not engage in conversation because they were not alert, attentive, calm and/ or coherent. Impaired mobility on presentation was defined as lack of a stable independent gait. Therefore, any patients that were unsteady on their feet, needed a walking stick or other aid to steady themselves, needed help to walk or were bedridden were considered to have an impaired mobility. Oxygen saturation and heart rate were measured from the finger by the Acc $U$ Rate CMS 500D oximeter (CMS Mobility, Stafford, USA). A period from 30 to 60 seconds was required to obtain a stable pulse and oxygen saturation reading.

\section{Statistical methods and data analysis}

The disposition score awarded one point for altered mental status, one point for impaired mobility and one point for an oxygen saturation $<94 \% .{ }^{18}$ Numeric variables were compared using Student's t test and categorical variables were compared using chi squared analysis with Yates' continuity correction, when applicable; calculations were performed using Epi Info, version 6.0 (Centres for Disease Control and Prevention, Atlanta, USA). The $p$ value for statistical significance was 0.05 . The optimal cut-off to convert continuous variables into a categorical variable was the value with the highest chi squared. Adjustment of odds ratios was performed using LOGISTIC software. ${ }^{21}$ The $C$ statistic was used to assess the discrimination of the score for hospital admission according to the method of Hanley and McNeil. ${ }^{22}$

\section{Ethics}

Ethical approval of the study was obtained from the scientific committee at Kitovu Hospital. The study conforms to the principles outlined in the Declaration of Helsinki. ${ }^{23}$ The study is reported in accordance with the STROBE statement. ${ }^{24}$

\section{Results}

From 23 November 2020 to 30 March 2021, 5,334 patients attended the hospital's emergency and outpatient departments, and $646(12.1 \%)$ were admitted: $176(27.2 \%)$ required surgery and the remainder required medical treatment. The five patients who died in the emergency department all had a score of 3 points. Half of all patients $(2,667)$ were seen before 25 January 2021 and the other half were seen after this date. Patients seen before 25 January 2021 were older, had lower oxygen saturations and were 
Table 1. Variables examined

\begin{tabular}{|c|c|c|c|c|}
\hline & $\begin{array}{l}\text { All patients, } \\
n=5,334\end{array}$ & $\begin{array}{l}\text { Before } 25 \text { January 2021, } \\
n=2,667\end{array}$ & $\begin{array}{l}\text { After } 25 \text { January 2021, } \\
n=2,667\end{array}$ & $p$ value \\
\hline Age, years (median; IQR) & 44.8 SD $19.8(41 ; 28-50)$ & 45.6 SD 20.1 (42; 28-61) & 44.1 SD $19.5(40 ; 28-58)$ & 0.006 \\
\hline Heart rate, bpm (median; IQR) & 73.0 SD 19.5 (68; 60-78) & 73.5 SD $19.1(68 ; 60-79)$ & 72.5 SD $19.9(67 ; 60-77)$ & 0.09 \\
\hline Oxygen saturation, \% (median; IQR) & 96.4 SD 4.9 (98; 95-99) & 96.0 SD 5.7 (97; 95-99) & 96.8 SD 3.9 (98; 95-99) & $<0.00001$ \\
\hline Age $\geq 57$ years, $n(\%)$ & $1,554(29.1)$ & $832(31.2)$ & $722(27.1)$ & 0.001 \\
\hline Male, $n(\%)$ & $2,099(39.4)$ & $1,072(40.2)$ & $1,027(38.5)$ & 0.22 \\
\hline Heart rate $>84 \mathrm{bpm}, \mathrm{n}(\%)$ & $994(18.6)$ & $523(19.6)$ & $471(17.7)$ & 0.07 \\
\hline Altered mental status, $\mathrm{n}(\%)$ & $912(17.1)$ & $457(17.1)$ & $455(17.1)$ & 0.97 \\
\hline Impaired mobility, $\mathrm{n}(\%)$ & $1,069(20.0)$ & $571(21.4)$ & $498(18.7)$ & 0.01 \\
\hline Oxygen saturation $<94 \%, n(\%)$ & $643(12.1)$ & $381(14.3)$ & $262(9.8)$ & $<0.00001$ \\
\hline Triage score of zero points, $n(\%)$ & $3,876(72.7)$ & $1,860(70.5)$ & $1,996(74.8)$ & 0.0004 \\
\hline Patients admitted, n (\%) & $646(12.1)$ & $380(14.2)$ & $266(10.0)$ & $<0.00001$ \\
\hline
\end{tabular}

more likely to have impaired mobility, and more were admitted to hospital (14.2\% versus $10.0 \%$; $p<0.0001$; Table 1 ).

On average, 42 patients per day were assessed: 42.3 patients per day for the 63 days before 25 January 2021 and 41.7 patients per day for the 64 days after 25 January 2020; 6.0 patients per day were admitted before 25 January 2021 and 4.2 patients per day were admitted after 25 January 2021. Patients who were admitted were older ( 50.7 years SD 21.8 vs 44.0 years SD 19.4; $p<0.00001$ ), had faster heart rates ( 84.5 beats per minute SD 25.6 vs 71.4 beats per minute SD 18.0; $p<0.00001)$ and had lower oxygen saturations ( $91.6 \%$ SD 9.3 vs $97.0 \%$ SD 3.5; $p<0.00001$ ). An age of $\geq 57$ years and a heart rate of $>84$ beats per minute had the odds ratios for admission with the highest chi squared values. Admission to hospital was also associated with mental status, mobility, oxygen saturation, heart rate, gender, and age, and half were assessed before 25 January 2021 and half after. The highest odds ratios for admission, both unadjusted and adjusted, were associated with the three components of the disposition score (mental status, gait and oxygen saturation; Table 2). The C statistic for the discrimination of the score for admission was 0.953 (95\% confidence interval (CI) 0.941-0.964).
Most patients (73\%) had a disposition of score of zero points and only 25 of these patients ( $0.6 \%)$ were admitted; 593 patients had one point (5.2\% admitted), 564 had two points (58.0\% admitted) and 301 had three points ( $87.4 \%$ admitted). A score $\geq 1$ point had a sensitivity for admission of $96.1 \%$, a specificity of $82.1 \%$, positive predictive value of $42.5 \%$, a negative predictive value of $99.4 \%$, a positive likelihood ratio of 5.36 and a negative likelihood ratio of 0.05 . Only 301 (5.6\%) patients had a score of 3 points and $263(87.4 \%)$ were admitted, there were fewer patients with 3 points admitted after 25 January 2021 ( $79 \%$ vs $94 \%$; $p=0.003)$. There were other statistically significant differences between the proportion of patients admitted according to the disposition score before and after 25 January 2021 (Fig 1). Despite these differences, there was no statistically significant difference between the $\mathrm{C}$ statistic for the discrimination of the disposition score for admission for patients assessed before and after 25 January 2021 (0.947 (95\% CI 0.932-0.963) vs 0.963 (95\% CI $0.946-0.978) ; p=0.16)$.

Of the 301 patients with a disposition score of three points, 38 $(12.6 \%)$ were not admitted, five of whom died in the emergency department; the remaining 33 patients were significantly older

Table 2. Admission variables odds ratios assessed

\begin{tabular}{|c|c|c|c|c|c|c|}
\hline Variable & $\mathbf{n}$ & Admitted & $\begin{array}{l}\text { Unadjusted odds } \\
\text { ratio }(95 \% \mathrm{CI})\end{array}$ & $\mathrm{p}$ & $\begin{array}{l}\text { Adjusted odds } \\
\text { ratio }(95 \% \mathrm{CI})^{\mathrm{a}}\end{array}$ & $\mathrm{p}$ \\
\hline Altered mental status & 912 & $65.1 \%$ & $157.0(114.2-216.4)$ & $<0.00001$ & $30.3(20.3-45.3)$ & $<0.00001$ \\
\hline Impaired mobility & 1,069 & $55.4 \%$ & $96.8(71.2-131.8)$ & $<0.00001$ & $9.8(6.5-14.7)$ & $<0.00001$ \\
\hline Oxygen saturation $<94 \%$ & 643 & $44.8 \%$ & $9.8(8.1-11.9)$ & $<0.00001$ & $4.9(3.5-6.8)$ & $<0.00001$ \\
\hline Heart rate $>84 \mathrm{bpm}$ & 994 & $28.6 \%$ & $4.4(3.7-5.3)$ & $<0.00001$ & $2.0(1.5-2.7)$ & $<0.00001$ \\
\hline Age $\geq 57$ years & 1,554 & $18.0 \%$ & $2.0(1.7-2.4)$ & $<0.00001$ & $0.6(0.4-0.7)$ & 0.0001 \\
\hline Male & 2,099 & $16.6 \%$ & $2.0(1.7-2.3)$ & $<0.00001$ & $2.0(1.5-2.6)$ & $<0.00001$ \\
\hline Seen after 25 January 2021 & 2,667 & $10.0 \%$ & $0.7(0.6-0.8)$ & $<0.00001$ & $0.4(0.3-0.5)$ & $<0.00001$ \\
\hline
\end{tabular}




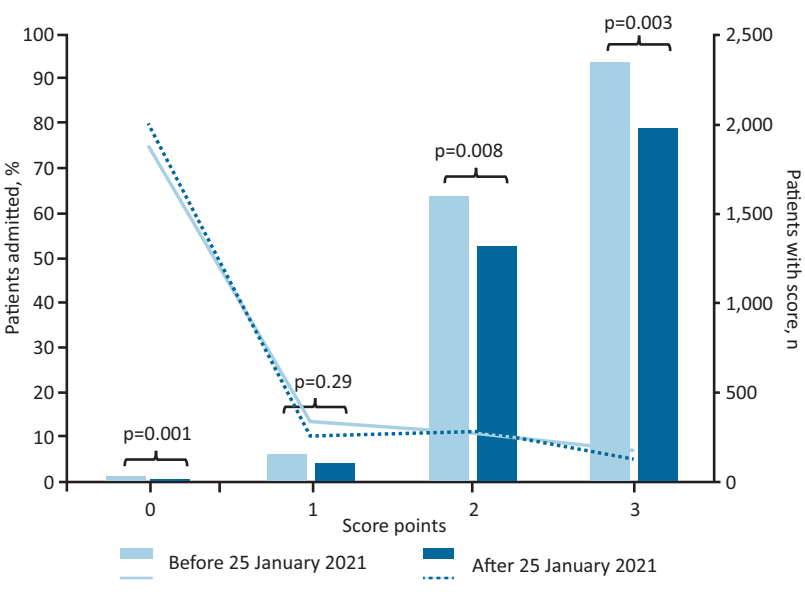

Fig 1. Patients admitted by score and date of presentation.

(74.4 years SD 21.3 vs 56.8 years SD 20.5; $p<0.00001$ ) and had higher oxygen saturations than patients with a score of three points who were admitted $(90.2 \%$ SD 3.9 vs $86.3 \%$ SD $8.6 ; p=0.01)$.

\section{Discussion}

\section{Main findings}

This paper shows that the decision to admit patients to hospital in a low-resource setting is strongly associated with their mental status, mobility and oxygen saturation on arrival; although other factors were identified, these three factors alone have a high discrimination for hospital admission.

\section{Limitations}

This small study was performed in a single centre. Although we believe that joint outpatient and emergency departments are common in low-resource settings, it is uncertain how the acuity and illness severity of the patients studied compares with other units. Moreover, we did not record or consider the number of patients who attended repeatedly or who had chronic conditions. For the study to be practical and affordable, only a small number of variables were examined; many other factors that were not examined may also influence hospital admission decisions.

The assessment of whether a patient was alert, attentive, calm and coherent was subjective. Despite this, the number of patients deemed to have normal mental status remained consistent at $83 \%$ throughout and in both phases of the study. More objective assessments of mental status (such as the months backwards test) are currently being examined. ${ }^{25}$ Some patients with impaired mobility may have had a temporary trivial impairment, such as a twisted ankle. However, such patients usually do not need help to maintain stability and nearly all can maintain their independence. Therefore, in practice there was no difficulty in identifying patients who had a stable independent gait and did not require assistance to mobilise. Despite concern about the accuracy of oximeters in patients with dark skin, our study confirms oximetry's clinical value. ${ }^{26}$ In the past, it has been suggested that oximetry is unaffordable in low-resource settings but this is no longer true: cheap ( US\$30), accurate and robust pulse oximeters are now widely available. ${ }^{27}$ Thanks to a recent donation from Electric Aid Ireland, a limited number of oximeters were available to clinical staff during our study. However, many clinicians are not accustomed to them and may have not relied on them to make clinical decisions.

\section{Interpretation}

Other factors that might influence hospital admission include severe pain and injury, the number of beds available, the likely benefit of hospital treatment, and if the patient wants it and can afford to pay for it. None of these were examined in this study. Nevertheless, our results suggest that admission decisions made in this low-resource setting were mostly influenced by three clinical factors and less influenced by non-clinical factors than decisions made in high-income countries.

Patients seen before 25 January 2021 were significantly older, more likely to be hypoxic and have impaired mobility, which may explain why more of them were admitted to hospital. However, it is also possible that criteria for admission varied between physicians. The difference in age between patients with a score of three points who were and were not admitted suggests that, for many older patients, hospital admission may have been considered futile. We are also concerned that some patients declined admission because they could not afford to pay for it. These issues need to be further studied.

Since the hospital has 85 medical and surgical beds and an average length of stay of 4.5 days SD 3.7 (unpublished data), more than 18 admissions per day would be needed to achieve $100 \%$ bed capacity. Therefore, bed availability is unlikely to account for any variation in the admission rate. It is also unlikely that the Hawthorne effect can explain any change in admission practice observed after 25 January 2021; the observations recorded on patient presentation were not shared with any clinicians, and disposition scores were not calculated until the final data analysis performed after the study was completed. ${ }^{28}$ The change in practice after 25 January 2021 might be explained by the ongoing COVID-19 pandemic. Unfortunately, the number of diagnostic tests available in the hospital was limited, and we cannot state with certainty how many of the patients observed during the study were infected with SARS-CoV-2.

\section{Clinical relevance}

This study was intended to demonstrate that the disposition score was practical and compatible with current admission practice; we did not wish to introduce a score that would produce an unrealistic increase in hospital admissions, or any further burden on the hospital's limited resources. We did not expect our score to identify patients who were going to be admitted to hospital as well as it did with such a high discrimination. ${ }^{29}$ However, Cameron et al have also developed a simple score in Glasgow that accurately predicted the likelihood of admission at the time of triage. ${ }^{14}$ Although their score did not consider mobility, it contained only six other variables: the patients' triage category, age, their vital signs as captured by the National Early Warning Score, how the patient arrived at the hospital, their referral source and history of prior admissions. The score also had excellent discrimination with a $\mathrm{C}$ statistic for hospital admission of 0.877 (95\% CI 0.875-0.880).

Our study confirms our score to be simple and practical. In acute situations, when many variables must be considered, 
simple scores may predict better than expert intuition as they consistently apply the same rules and ignore irrelevant detail. ${ }^{30}$ This study suggests the score can rapidly and accurately identify those patients who are the least and most likely to be admitted. It is reasonable to assume that those presenting with a score of zero points who do not have severe pain or injury (ie most patients) are unlikely to suffer if they wait to be seen. In contrast, eventual admission to hospital is likely for the minority of patients who have high scores, and these patients will benefit most from immediate attention.

\section{Conclusion}

In a low-resource setting, a simple score based on mental status, mobility and oxygen saturation identified those outpatient and emergency department patients who were most and least likely to be subsequently admitted to hospital with a high degree of discrimination.

\section{Summary}

\section{What is known?}

There is considerable variability in the number of emergency patients admitted to hospital.

\section{What is the question?}

What factors influence the decision to admit patients?

\section{What was found?}

In a low-resource setting, mental status, mobility and oxygen saturation rapidly identified patients most and least likely to be admitted to hospital.

\section{What is the implication?}

A simple score rapidly identifies patients who need hospital admission, and its use may reduce waiting time for these patients.

\section{Acknowledgements}

We wish to acknowledge the donation of oximeters by Electric Aid Ireland, and Tapa Healthcare DAC (Dundalk, Ireland) for the complimentary use of their Rapid Electronic Assessment Data System (READS). We would also like to thank Mr Alan Murray, Passage West, Ireland, for his generous IT support.

\section{Conflicts of interest}

John Kellett is a major shareholder, director and chief medical officer of Tapa Healthcare DAC.

\section{References}

1 Hunter AEL, Spatz ES, Bernstein SL, Rosenthal MS. Factors influencing hospital admission of non-critically ill patients presenting to the emergency department: a cross-sectional study. J Gen Intern Med 2016;31:37-44.

2 National Institute for Health and Care Excellence. Emergency and acute medical care in over 16s: service delivery and organisation: NICE guideline [NG94]: Chapter 21, Standardised criteria for hospital admission. NICE, 2018. www.ncbi.nlm.nih.gov/books/ NBK564899

3 National Audit Office. Emergency admissions to hospital: managing the demand. NAO, 2013. www.nao.org.uk/wp-content/
uploads/2013/10/10288-001-Emergency-admissions.pdf [Accessed 7 April 2021].

4 Burch VC, Tarr G, Morroni C. Modified early warning score predicts the need for hospital admission and inhospital mortality. Emerg Med J 2008;25:674-8.

5 Pope I, Burn H, Ismail SA, Harris T, McCoy D. A qualitative study exploring the factors influencing admission to hospital from the emergency department. BMJ Open 2017;7:e011543.

6 O'Cathain A, Knowles E, Maheswaran R et al. A system-wide approach to explaining variation in potentially avoidable emergency admissions: national ecological study. BMJ Qual Saf 2014:23:1-9.

7 Twomey M, Wallis LA, Myers JE. Limitations in validating emergency department triage scales. Emerg Med J 2007;24:477-9.

8 Hing E, Bhuiya F. Wait time for treatment in emergency departments: 2009. NCHS Data Brief 2012:1-8.

9 Guttmann A, Schull MJ, Vermeulen M], Stukel TA. Association between waiting times and short-term mortality and hospital admission after departure from emergency department: population-based cohort study from Ontario, Canada. BMJ 2011;342:d2983.

10 Levine SD, Colwell CB, Pons PT et al. How well do paramedics predict admission to the hospital? A prospective study. J Emerg Med 2006;31:1-5.

11 Kosowsky JM, Shindel S, Liu T, Hamilton C, Panciolli AM. Can emergency department triage nurses predict patients' dispositions? Am J Emerg Med 2001;19:10-14.

12 Brillman JC, Doezema D, Tandberg D et al. Triage: Limitations in predicting need for emergent care and hospital admission. Ann Emerg Med 1996;4:493-500.

13 Beardsell I, Robinson S. Can emergency department nurses performing triage predict the need for admission? Emerg Med J 2011;28:959-62.

14 Cameron A, Rodgers K, Ireland A, Jamdar R, McKay GA. A simple tool to predict admission at the time of triage. Emerg Med J 2015;32:174-9.

15 Purdy S. Avoiding hospital admissions: what does the evidence say. London: The King's Fund, 2010.

16 Leegon J, Jones I, Lanaghan K, Aronsky D. Predicting hospital admission in for emergency department patients using a Bayesian network. AMIA Annu Symp Proc 2005;2005:1022.

17 Leegon J, Jones I, Lanaghan K, Aronsky D. Predicting hospital admission in a pediatric emergency department using an artificial neural network. AMIA Annu Symp Proc 2006;2006:1004.

18 Wasingya-Kasereka L, Nabatanzi P, Nakitende I et al; for the Kitovu Hospital Study Group. Two simple replacements for the Triage Early Warning Score to facilitate the South African Triage Scale in low resource settings. Afr J Emerg Med 2021;11:53-9.

19 The World Bank. World Bank Country and Lending Groups. The World Bank, 2020. https://datahelpdesk.worldbank.org/knowledgebase/ articles/906519-world-bank-country-andlendinggroups [Accessed 04 May 2020].

20 Mullan F, Frehywot S. Non-physician clinicians in 47 sub-Saharan African countries. Lancet 2007;370:2158-63.

21 Dallal GE. LOGISTIC: a logistic regression program for the IBM PC. Am Stat 1988;42:272.

22 Hanley JA, McNeil BJ. A method of comparing the areas under receiver operating characteristic curves derived from the same cases. Radiology 1983;148:839-43.

23 World Medical Association. World Medical Association Declaration of Helsinki: ethical principles for medical research involving human subjects. JAMA 2013;310:2191-4.

24 Vandenbroucke JP, von Elm E, Altman DG et al. Strengthening the Reporting of Observational Studies in Epidemiology (STROBE): explanation and elaboration. Epidemiology 2007;18:805-35.

25 Hasemann W, Grossmann FF, Bingisser R et al. Optimizing the month of the year backwards test for delirium screening of 
older patients in the emergency department. Am J Emerg Med 2019;37:1754-7.

26 Sjoding MW, Dickson RP, Iwashyna T], Gay SE, Valley TS. Racial bias in pulse oximetry measurement. N Engl J Med 2020;383:2477-8.

27 Weber MW, Mulholland EK. Pulse oximetry in developing countries. Lancet 1998;351:1589.

28 McCarney R, Warner J, Iliffe S et al. The Hawthorne Effect: a randomised, controlled trial. BMC Med Res Methodol 2007;7:30.
29 Hosmer DW, Lemeshow S. Applied logistic regression, 2nd edn: Chapter 5. New York: Wiley, 2000:160-4.

30 Kahneman D. Thinking, fast and slow. Macmillan, 2011.

Address for correspondence: Dr John Kellett, Hospital of South West Jutland, Finsensgade 35, 6700 Esbjerg, Denmark. Email: kellettjg@gmail.com 